\title{
Seeing the Invisible: Scanning Near-Field Ultrasound Holography (SNFUH) for High Resolution Sub-Surface Imaging
}

\author{
Gajendra Shekhawat ${ }^{*}$ and Vinayak P. Dravid ${ }^{\# *}$ \\ NUANCE Center, *International Institute for Nanotechnology, 1161 Cook Hall, Northwestern \\ University, Evanston, IL 60208 \\ \# Material Science and Engineering, 1141 Cook Hall, Northwestern University, Evanston, IL
}

We have developed a novel scanning near-field ultrasound holography (SNFUH) technique which combines the nanometer-scale spatial resolution of conventional scanning probe microscope (SPM) with the surface and subsurface imaging capabilities [1]. This technique fills the critical void in characterization and investigation of the static and dynamic mechanics of nanoscale systems and address emerging issues in imaging and analysis of diverse "embedded" nano and microscale structures, and engineered systems. The SNFUH development integrates three major approaches: a unique combination of scanning probe microscope platform (which enjoys excellent lateral and vertical resolution) coupled to microscale ultrasound source and detection (which facilitates "looking" deeper into structures, section-by-section) and a novel holography approach (to enhance phase resolution and phase coupling in imaging).

Existing far-field ultrasonic or acoustic microscopy techniques [2] suffer from spatial resolution limitations. Moreover, all far field microscopies lack: resolution Limitations due to Rayleigh limit: $0.51 \lambda /$ N.A., coupling fluid attenuation $\sim \mathrm{f}^{2}$, and impedance mismatches. Recently ultrasonic force microscopy [3-4] has been widely used to map the elastic properties of soft and hard surfaces.

In SNFUH approach, a high frequency ( 100's of KHz to several MHz) acoustic wave is launched from the bottom of the specimen, while another wave is launched on the AFM cantilever, albeit at a slightly different frequency. The interference of these two waves would nominally form so-called "beats" and "product frequencies" which is monitored by the AFM tip, which itself acts as an antenna for both phase and amplitude of the beats and product frequencies. As the specimen acoustic wave gets perturbed by sub-surface (and surface) features, especially its phase, the local acoustic interference is very effectively monitored by the AFM tip. Thus, within the near-field regime (which enjoys superb lateral and vertical resolution), the acoustic wave (which is nondestructive and sensitive to mechanical/elastic variation in its "path") is fully analyzed, point-bypoint, by the AFM acoustic antenna in terms of phase and amplitude.

The efficacy of SNFUH for in-vitro biological imaging and indentifying the buried structures is demonstrated on acoustically active liposomes (AAL) which contains small amount of air. AAL have oil layer in which therapeutics can be dissolved, the lipid shell and the air-bubbles interior. These liposomes have potential to carry drugs, ease of conjugation with antibodies, peptides and their acoustic activity could enable them to respond to ultrasound stimulation by releasing their contents and has been reported elsewhere [5]. Figure 1 depicts a high resolution and remarkably high contrast from AAL, and shows embedded air-bubbles embedded in them. Here, we demonstrated direct and real-space in-vitro imaging of the presence of air-bubbles and proteins inside liposome's without any labels or sectioning of cells, and under physiologically viable conditions. Figure 2 shows another potential application of SNFUH approach in direct imaging of 
buried magnetic nanoparticles inside silica shells. Cobalt magnetic particles from 15-25 nm are enclosed in silica core shell. The presentation will cover the conceptual development of SNFUH and emerging applications in microelectronic defect analysis and cell biology. [6]

\section{References}

[1] G.Shekhawat and Vinayak Dravid, Science 310, 89 (2005)

[2] G. A. D. Briggs, Acoustic Microscopy, (Clarendon Press, Oxford, 1992) p. 28.

[3] O. V. Kolosov, K.Yamanaka, Jpn. J. Appl. Phys. 32, 1095 (1993).

[4] G.S.Shekhawat et al. Nanoscale elastic imaging of Aluminum/low-k dielectric interconnect structure, Material Research Society Symposium Proceedings, Vol. 612 (2001) pp.1

[5] S. Huang and R. MacDonald, Biochimica et Biophysica Acta 1665, 134-141 (2004).

[6] The SNFUH measurements were carried out in the NIFTI facility of NUANCE Center at Northwestern University. NUANCE Center is supported by NSF-NSEC, NSF-MRSEC, Keck Foundation, the State of Illinois, and Northwestern University. We thank M. Aslam for silica core shell particles samples and Dr. Robert Macdonald and S. Huang for Liposomes samples.

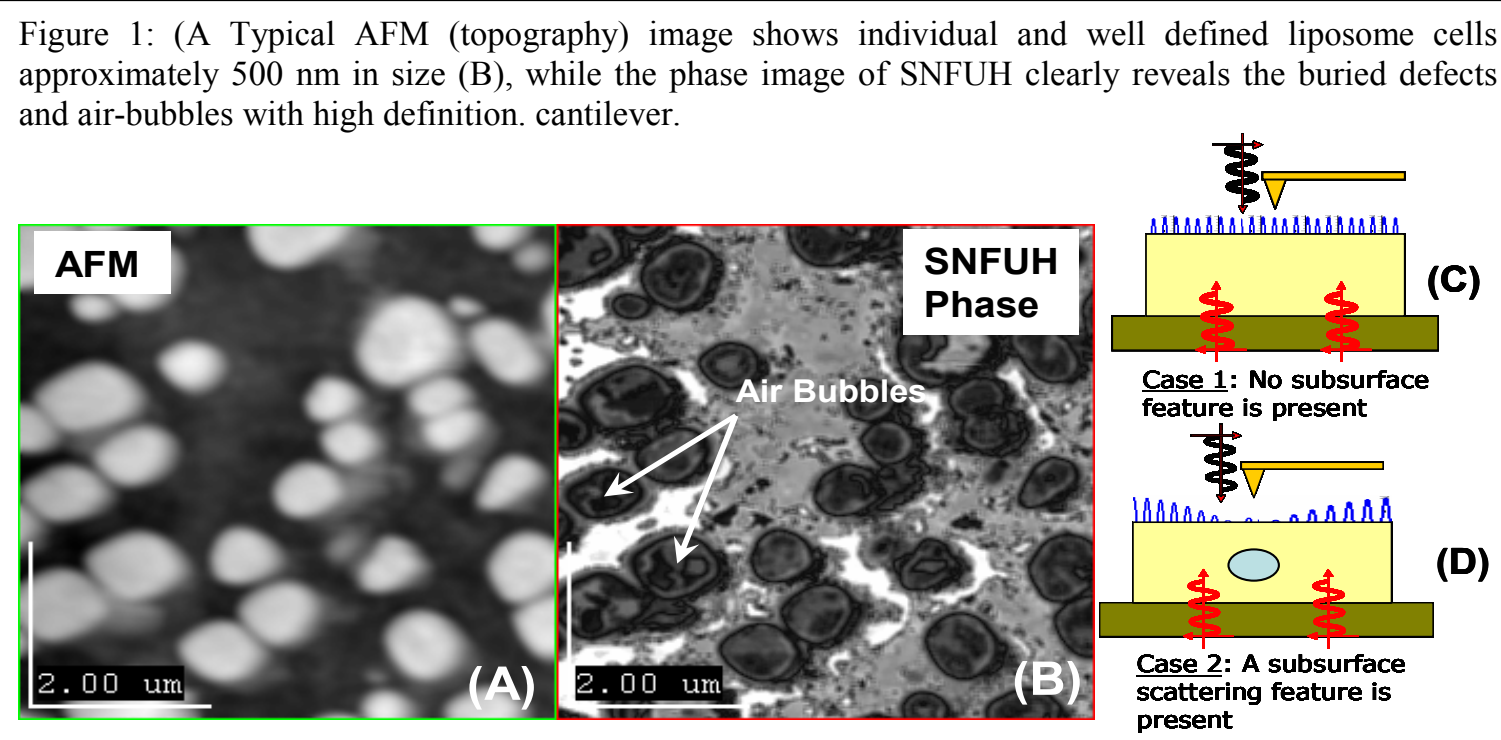

Figure 2: (A) AFM topography and (B) SNFUH phase images from magnetic nanoparticles enclosed in silica core shell. Topography shows typical surface features silica core shell spread uniformly on mica substrate, while SNFUH phase image demonstrates remarkable contrast from magnetic nanoparticles enclosed in core shell at nanoscale spatial resolution.

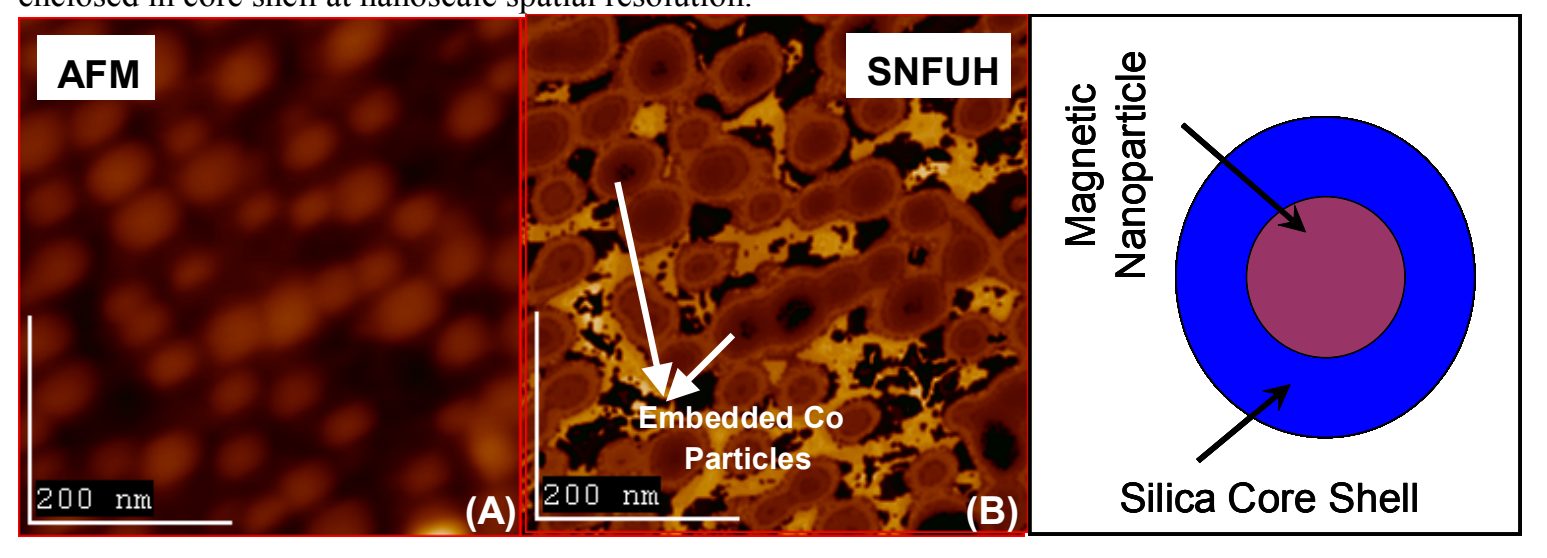

